\title{
Development of Novel Flow Chamber to Study Endothelial Cell Morphology: Effects of Shear Flow with Uniform Spatial Gradient on Distribution of Focal Adhesion*
}

\author{
Daisuke YOSHINO**, , Naoya SAKAMOTO***,,$\dagger$, Keita TAKAHASHI****, \\ Eri INOUE** and Masaaki SATO**,*** \\ ${ }^{* *}$ Graduate School of Biomedical Engineering, Tohoku University \\ 6-6-01 Aramaki-Aoba, Aoba, Sendai 980-8579, Japan \\ E-mail: yoshino@plasma.ifs.tohoku.ac.jp \\ ${ }^{* * *}$ Graduate School of Engineering, Tohoku University \\ ****School of Engineering, Tohoku University \\ Present Address: ${ }^{\dagger}$ Institue of Fluid Science, Tohoku University \\ ${ }^{\mathrm{t \dagger}}$ Kawasaki University of Medical Welfare
}

\begin{abstract}
Fluid shear stress (SS) is well known to cause morphological changes in vascular endothelial cells (ECs) accompanied by alteration in actin cytoskeletal structure and distribution of focal adhesions. Recent studies have shown that spatial SS gradient also has effects on EC morphology, but the detailed mechanisms of EC responses to SSG remain unclear. In the present study, we sought morphological responses of ECs under SS and uniform SSG condition using a newly developed flow chamber. Confluent ECs were exposed to SS with SSG for 24 hours. Focal adhesions of the EC under SS without SSG were localized in the cell periphery. In contrast, focal adhesions were expressed not only in the periphery but also in interior portion of cells after exposure to SS with SSG. Unlike ECs exposure to SS developed thick actin filaments aligned to the direction of flow no development of thick actin filaments but thin and short filaments were observed in ECs after 24-hour exposure to SS with SSG. Since the distribution of focal adhesion is of critical importance for development of actin filaments and cell morphological changes, these results suggest that SSG suppresses redistribution of focal adhesions, resulting in the inhibition of EC morphological changes and development of thick actin filaments in response to flow.
\end{abstract}

Key words: Endothelial Cells, Shear Stress, Shear Stress Gradient, Focal Adhesion, Cytoskeleton

\section{Introduction}

Vascular endothelial cells (ECs), lining on the luminal wall of blood vessels, change their morphology and regulate a variety of physiological functions of vessel walls in response to hemodynamic stimuli such as fluid shear stress (SS) in vivo. There have been many previous studies that have investigated responses of ECs to SS and shown that ECs elongate and align to the direction of flow in response to physiological levels of SS (1 2 Pa)

(1). Previous studies have also shown that magnitude of SS influences EC morphology and 
may provoke various cellular functions that play important roles in vascular homeostasis ${ }^{(2,}$ 3).

It was also found that cytoskeleton and focal adhesion have important roles in EC morphology. A number of actin filaments, which is one of the cytoskeletons, grow to actin stress fibers aligned to the direction of flow in ECs exposed to SS ${ }^{(4)}$. Focal adhesion is the protein complex through which the cytoskeletons connect to extracellular matrix. This complex consists of integrin, vinculin, talin, paxilin, focal adhesion kinase (FAK), and so on. Paxillin is particularly thought to play an important role in responses of ECs to mechanical stimuli. In a EC exposed to fluid flow, there is difference in phosphorylated state of paxillin between upstream and downstream within the cell ${ }^{(5)}$, suggesting that differences in the expression level and phosphorylated state of paxillin within an EC influence morphological changes of ECs.

Recently, spatial SS gradient (SSG) has also attracted a great deal of attention as a key factor for EC morphology under flow conditions. Szymanski et al. ${ }^{(6)}$ exposed SS (0 20.6 $\mathrm{Pa})$ and SSG (-9 14 $\mathrm{Pa} / \mathrm{mm})$ using a T-shaped flow chamber mimicking arterial bifurcations, and showed that ECs density was increased at the high SS and high SSG region. Sakamoto et al. exposed high SS $(\sim 16 \mathrm{~Pa})$ and high SSG $(\sim 34 \mathrm{~Pa} / \mathrm{mm})$ using the T-chamber, and showed SSG suppressed the orientation of ECs ${ }^{(7)}$. However, it is too complicated to evaluate effects of SSG on EC morphology by using T-chamber because it is very difficult to control the combination of SS and SSG in the T-chamber. Although a recent study developed a flow chamber which has converging and diverging channel to create well-defined SSG condition ${ }^{(8)}$, the magnitude of SSG in the chamber was much lower compared with the previous studies, and knowledge of morphological responses of ECs to combination of SS and SSG is still limited.

In the present study, we develop a novel flow chamber, which can generate uniform spatial SS gradient, for evaluating effects of SSG on endothelial morphology. Using the developed chamber, we observe changes in cytoskeletal structures and distribution of focal adhesions of ECs, and discuss the effect of SSG on morphology of ECs.

\section{Design of novel flow chamber for generating uniform shear stress gradient} (9),

SS $(=\tau)$ occurring in a parallel-plate flow chamber is defined by the following equation

$$
\tau=\frac{6 Q \mu}{w h^{2}}
$$

where $Q$ is flow rate, $\mu$ is fluid viscosity of culture medium, $w$ is width of a flow channel, and $h$ is a channel height. Equation (1) is also valid for Newtonian fluid in fully developed laminar flow in a flow channel with a high aspect ratio $(w>>h)^{(8)}$. Therefore, we considered a flow channel with a wide and low roof in a newly designed flow chamber as illustrated in Fig. 1A. The flow channel consists of three regions: an "entrance" region, a "gradient" region, and an "exit" region. The length of each region is denoted as $L_{\mathrm{en}}, L_{\mathrm{g}}$, or $L_{\text {ex }}$. The heights of "entrance" and "exit" regions are denoted as $h_{0}$ and $t$, respectively. In addition, the channel height in the "gradient" region is defined by the function $h(x)$. Distance from the ideal origin of the function $h(x)$ to the start point of "gradient" region is indicated as $L_{0}$. By considering linear increase in $\mathrm{SS}, h(x)$ is set up as follow:

$$
h(x)=t \sqrt{\frac{L_{\mathrm{g}}+L_{0}}{x+L_{0}}} .
$$

SS and SSG $(=\mathrm{d} \tau / \mathrm{d} x)$ are given by following equations:

$$
\tau=\frac{6 Q \mu}{w t^{2}\left(L_{\mathrm{g}}+L_{0}\right)}\left(x+L_{0}\right),
$$




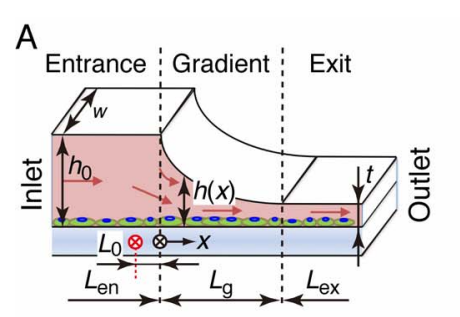

C

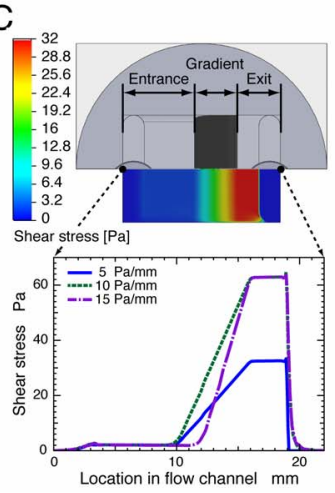

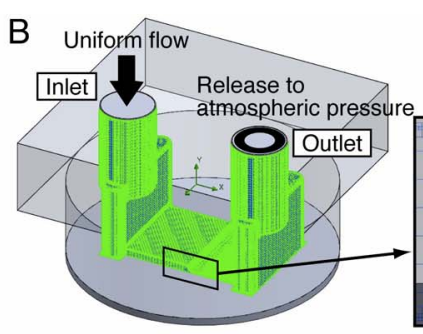

D

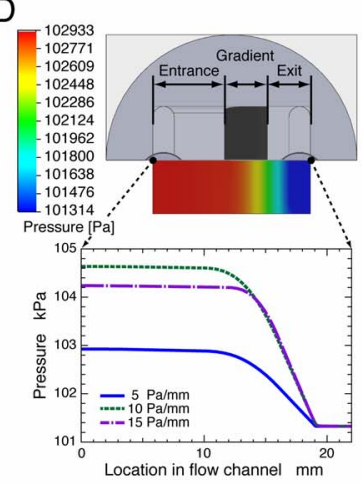

Fig. 1 The developed flow chamber that can generate uniform spatial SS gradient. (A) Schematic illustration of flow channel in the developed chamber. (B) Boundary conditions and the mesh model used for CFD simulation. (C) Simulated distribution of shear stress in flow channel. (D) Simulated distribution of pressure in flow channel.

$$
\frac{d \tau}{d x}=\frac{6 Q \mu}{w t^{2}\left(L_{\mathrm{g}}+L_{0}\right)} .
$$

The maximum and minimum SS values in the flow channel are obtained as follows:

$$
\begin{aligned}
& \left.\tau_{\max }\right|_{x=L_{\mathrm{g}}}=\frac{6 Q \mu}{w t^{2}}, \\
& \left.\tau_{\min }\right|_{x=0}=\frac{6 Q \mu L_{0}}{w t^{2}\left(L_{\mathrm{g}}+L_{0}\right)} .
\end{aligned}
$$

The length $L_{\mathrm{g}}$ of the "gradient" region and $L_{0}$ are derived by using above equations.

$$
\begin{aligned}
& L_{0}=\tau_{\min } /\left(\frac{d \tau}{d x}\right) \\
& L_{\mathrm{g}}=\left(\tau_{\max }-\tau_{\min }\right) /\left(\frac{d \tau}{d x}\right)
\end{aligned}
$$

The lengths of "entrance" and "exit" regions can be arbitrarily defined within a range of the flow channel length, which depends on the size of a dish. Once a designer gives the value of maximum SS, minimum SS, and SSG, dimensions of the required flow chamber are determined. In the present study, a flow chamber designed using the theory described above is named as the "derivative constant" chamber (D chamber). We designed 3 types of D chambers that can generate SSG values of 5,10 , and $15 \mathrm{~Pa} / \mathrm{mm}$, respectively. Table 1 presents dimensions of main design parameters of the $\mathrm{D}$ chamber. The length $L_{\mathrm{en}}$ of "entrance" region was set up to be sufficient to develop a boundary layer before "gradient" region by using a computational fluid dynamics (CFD) simulation described below.

In order to confirm the performances of the D chambers, the CFD simulation was performed by the finite volume method in SolidWorks Flow Simulation (Dassault Systèmes SolidWorks Corporation). Culture medium perfused in the chamber was modeled with the continuity equation and the Navier-Stokes equation for an incompressible Newtonian fluid. The mass density and viscosity of culture medium were set to $1000 \mathrm{~kg} / \mathrm{m}^{3}$ and $7.4 \times 10^{-4} \mathrm{~Pa} \cdot \mathrm{s}$, respectively, and the flow rate was set to $259(5 \mathrm{~Pa} / \mathrm{mm}), 503(10 \mathrm{~Pa} / \mathrm{mm})$, or $402 \mathrm{ml} / \mathrm{min}$ 
Table 1 Dimensions of main design parameters of the D chamber

\begin{tabular}{|l|c|c|c|}
\hline Design Parameter & $5 \mathrm{~Pa} / \mathrm{mm}$ & $10 \mathrm{~Pa} / \mathrm{mm}$ & $15 \mathrm{~Pa} / \mathrm{mm}$ \\
\hline Length of "entrance" region $L_{\mathrm{en}}(\mathrm{mm})$ & 7 & 7 & 9 \\
\hline Length of "gradient" region $L_{\mathrm{g}}(\mathrm{mm})$ & 6 & 6 & 4 \\
\hline Length of "exit" region $L_{\mathrm{ex}}(\mathrm{mm})$ & 3 & 3 & 3 \\
\hline $\begin{array}{l}\text { Distance from the ideal origin of the } \\
\text { function } h(x) \text { to the start point of "gradient" } \\
\text { region } L_{0}(\mathrm{~mm})\end{array}$ & 0.40 & 0.20 & 0.13 \\
\hline Width of flow channel $w(\mathrm{~mm})$ & 15 & 15 & 12 \\
\hline Height of "entrance" region $h_{0}(\mathrm{~mm})$ & 0.80 & 1.11 & 1.11 \\
\hline Height of "exit" region $t(\mathrm{~mm})$ & 0.20 & 0.20 & 0.20 \\
\hline
\end{tabular}

(15 $\mathrm{Pa} / \mathrm{mm}$ ). The flow rate profile was defined as the uniform flow, and it applied to the inlet of flow channel as a boundary condition. The pressure at the outlet boundary was set to the atmospheric pressure. We used the mesh of hexahedron cells for CFD simulation, and the number of computational cells was 471906 (5 Pa/mm), $495216(10 \mathrm{~Pa} / \mathrm{mm})$, or 482401 (15 $\mathrm{Pa} / \mathrm{mm})$. The configurations for CFD simulation described above are summarized in Fig. 1B. The CFD results are shown in Fig. $1 \mathrm{C}$, D. The SS in the figure indicates the value on the center line of bottom surface in the flow channel. In the D chamber with $5 \mathrm{~Pa} / \mathrm{mm}$ SSG, SS increased from $2 \mathrm{~Pa}$ to $32 \mathrm{~Pa}$ while SS value in D chambers with 10 and $15 \mathrm{~Pa} / \mathrm{mm}$ increased from $2 \mathrm{~Pa}$ to $62 \mathrm{~Pa}$. It was confirmed that linear increase in SS could be kept within the "gradient" region excluding edges of the flow channel. The Reynolds number in the flow channel was $779(5 \mathrm{~Pa} / \mathrm{mm})$ or $1510(10 \mathrm{~Pa} / \mathrm{mm}$ and $15 \mathrm{~Pa} / \mathrm{mm})$, respectively. Performances of the $\mathrm{D}$ chambers could be confirmed by using CFD simulation. In this paper, the D chamber with $15 \mathrm{~Pa} / \mathrm{mm}$ was used for flow-exposure experiments because SSG could come to $15 \mathrm{~Pa} / \mathrm{mm}$ at the arterial bifurcation ${ }^{(10)}$.

\section{Materials and Methods}

\subsection{Cell culture}

Human carotid artery endothelial cells (HCtAECs; Cell Applications) were cultured with Medium 199 (Invitrogen) containing $20 \%$ heat-inactivated fetal bovine serum (SAFC Bioscience), $10 \mu \mathrm{g} / \mathrm{l}$ human basic fibroblast growth factor (AUSTRAL Biologicals), and $0.1 \%$ penicillin/streptomycin (Invitrogen). HCtAECs from 5th to 9 th passages were cultured in $35 \mathrm{~mm}$ diameter glass-based dish (Iwaki) pre-coated with $0.1 \%$ bovine gelatin (Sigma-Aldrich), and used for the experiments.

\subsection{Flow-exposure experiment}

The flow chamber was connected to a flow circuit comprising a roller pump (Master Flex) and two reservoirs, as illustrated in Fig. 2. The flow circuit was filled with the same medium as that used during cell culture. One reservoir was open to the atmosphere, and the culture medium in the flow circuit was equilibrated with $93 \%$ air and $7 \% \mathrm{CO}_{2}$ to maintain $\mathrm{pH}$ at 7.4. The system was maintained at $37^{\circ} \mathrm{C}$ with a temperature-controlled bath. For the experimental condition of SS with/without SSG, we used the newly designed flow chamber and a parallel-plate flow chamber ${ }^{(11)}$, respectively. We focused on the cells exposed to the following combinations of SS and SSG; SS $=2$ and $6 \mathrm{~Pa}$, and $\mathrm{SSG}=0$ and $15 \mathrm{~Pa} / \mathrm{mm}$. Distance from the start point of "gradient" region to the position of observation is $0 \mathrm{~mm}$ (SS $=2 \mathrm{~Pa})$ or $0.27 \mathrm{~mm}(\mathrm{SS}=6 \mathrm{~Pa})$ in case where $\mathrm{SSG}$ is $15 \mathrm{~Pa} / \mathrm{mm}$. The SS values were determined as levels of physiological and high shear conditions by reference to previous studies ${ }^{(12,13)}$. 

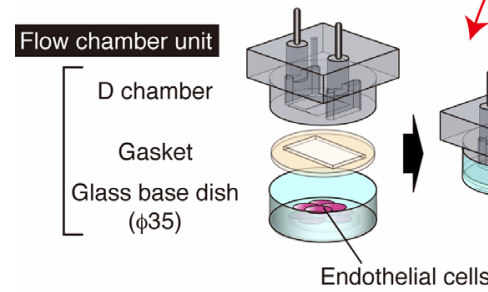

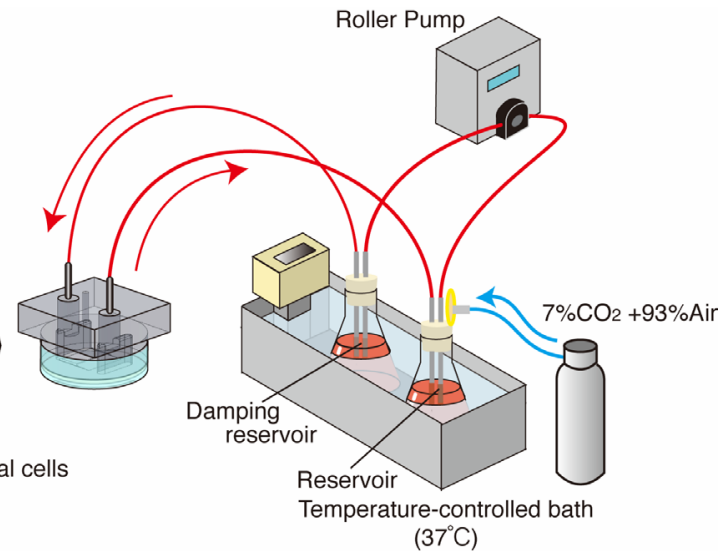

$\left(37^{\circ} \mathrm{C}\right)$

Fig. 2 Flow-exposure system consisting of a flow chamber, a roller pump, two reservoirs, and temperature-controlled bath.

\subsection{Fluorescent staining}

After 24-hour flow exposure, ECs were fixed with $4 \%$ paraformaldehyde phosphate buffer solution (Wako Pure Chemical Industries) for 15 minutes at room temperature and washed thrice with PBS (Dulbecco's PBS(-); Nissui Pharmaceutical). EC membranes were then permeabilized with $0.1 \%$ Triton X-100 (Wako Pure Chemical Industries) for 5 minutes. After washing thrice with PBS, ECs were then treated with Block Ace (DS Pharma Biomedical) for 45 minutes to prevent the non-specific adsorption of antibody. ECs were subsequently incubated with anti-mouse paxillin monoclonal antibody (BD Biosciences) and anti-rabbit VE-cadherin antibody (eBioscience) for an hour and a half, followed by secondary antibodies conjugated with anti-mouse Alexa Fluor 488 (Invitrogen) and anti-rabbit Alexa Fluor 633 (Invitrogen) for 1 hour. For staining nuclei and actin filaments, ECs were incubated with DAPI (Invitrogen) for 5 minutes, and Alexa Fluor 546 phalloidin (Invitrogen) for 20 minutes, respectively. Fluorescent images of paxillin, VE-cadherin, nuclei, and actin filaments were observed with an inverted confocal laser scanning microscope (Olympus).

\subsection{Evaluation of focal adhesion distribution}

It is important for evaluating localization of paxillin among the variously-sized ECs to normalize the sizes of the ECs. We firstly treated the fluorescent images to remove non-specific noises by using the discriminant analysis method. The outlines of ECs were extracted from the fluorescent images, and then the ellipsoids equivalent to ECs shapes were computed based on the extracted outlines by using ImageJ (National Institute of Health). Here, we achieved the central coordinate, lengths of major and minor axses, and orientation angle of the ellipsoid equivalent to the ECs. Then, areas and coordinates of paxillin in the extracted ECs were obtained by using the function of "Analyze Particles." The ellipsoids equivalent to the ECs were normalized to unit circles by using the dimensions of the ellipsoid. The coordinates of paxillin were converted from rectangular coordinates to polar coordinates, and we finally normalized coordinates of paxillin and trabscribed onto a unit circle. In addition, an expressed area of paxillin was highlighted with six levels of circular sizes.

\section{Results}

\subsection{Cell morphology and cytoskeletal structure}

Figure 3 shows fluorescent images of ECs after 24-hour flow exposure. ECs maintained 


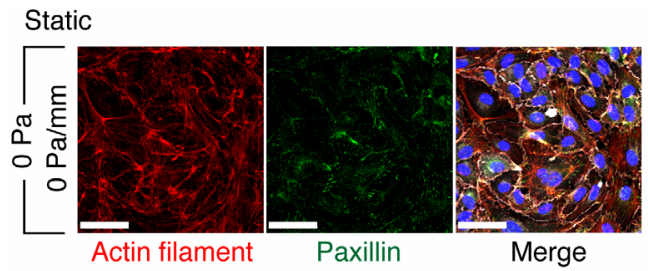

Flow, 24 hours
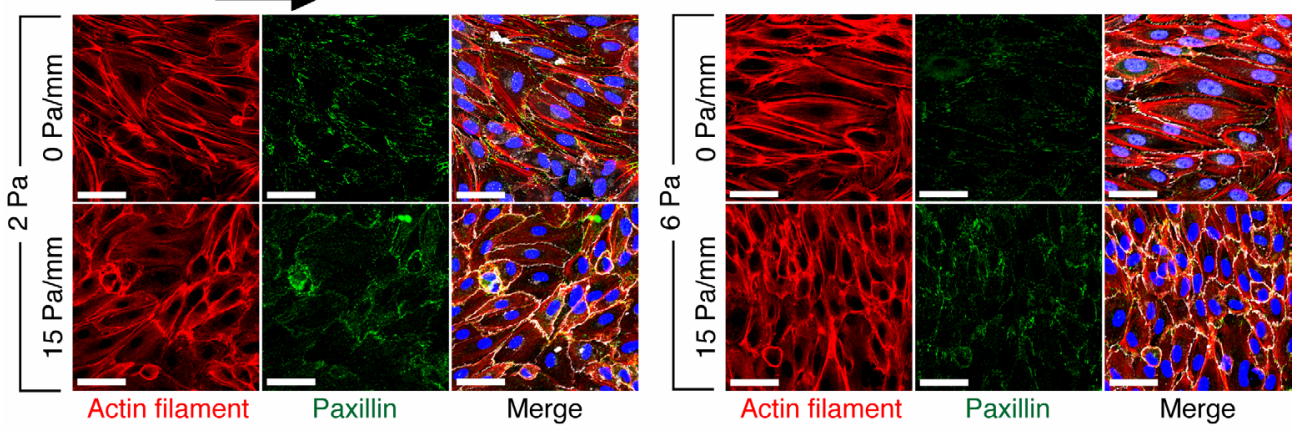

Fig. 3 Representative fluorescent images of ECs exposed to the combination of SS and uniform SSG for 24 hours. Red: actin filament, green: paxillin, bule: nucleus, white: VE-cadherin. "Merge" indicates the image merged with fluorescent images of actin filament, paxillin, nucleus, and VE-cadherin. Bar $=50 \mu \mathrm{m}$.

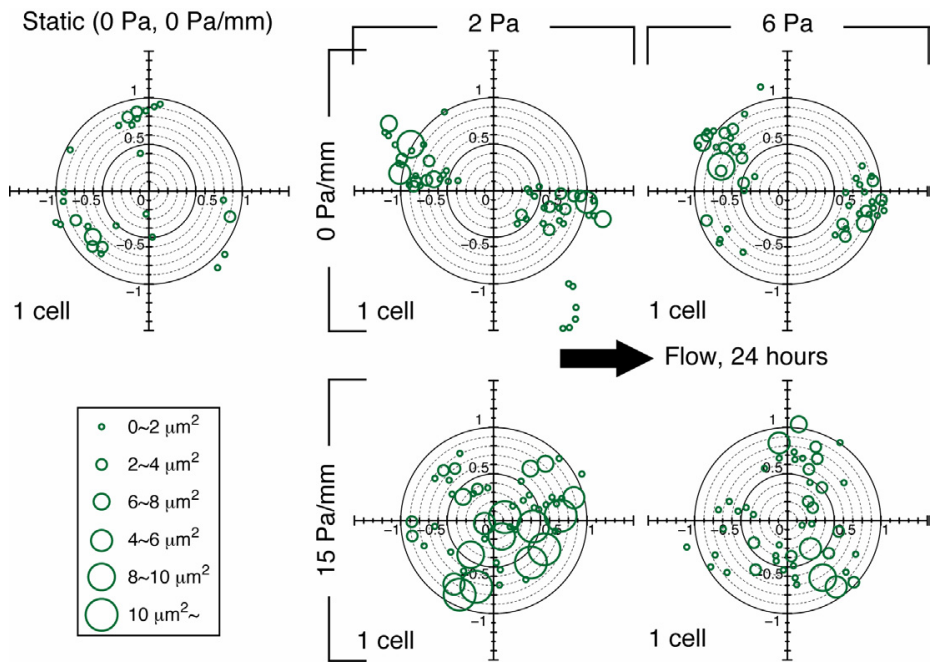

Fig. 4 Representative distributions of paxillin in ECs exposed to the combination of SS and uniform SSG for 24 hours.

a confluent monolayer, and formation of intercellular gaps, detachment, and damage were not observed in any of the experimental conditions. ECs exposed to SS without SSG elongated and oriented in the direction of flow. Actin filaments existing along the periphery of ECs developed and became thick. On the other hand, ECs under the condition of SS with SSG did not elongate and orient in the direction of flow. Actin filaments in the periphery of cells remained thin, and the thin and short filaments were formed in the interior portion of the cell.

\subsection{Distribution of paxillin in cell under shear stress with/without its spatial gradient}

Based on fluorescent images in Fig. 3, we evaluated distribution of paxillin in ECs by using the method described in section 3.4. The representative distribution of paxillin in an EC exposed to each flow condition is shown in Fig. 4. Figure 5 shows histograms obtained by counting the number of paxillin from the center to the periphery of an EC. Paxillin in 


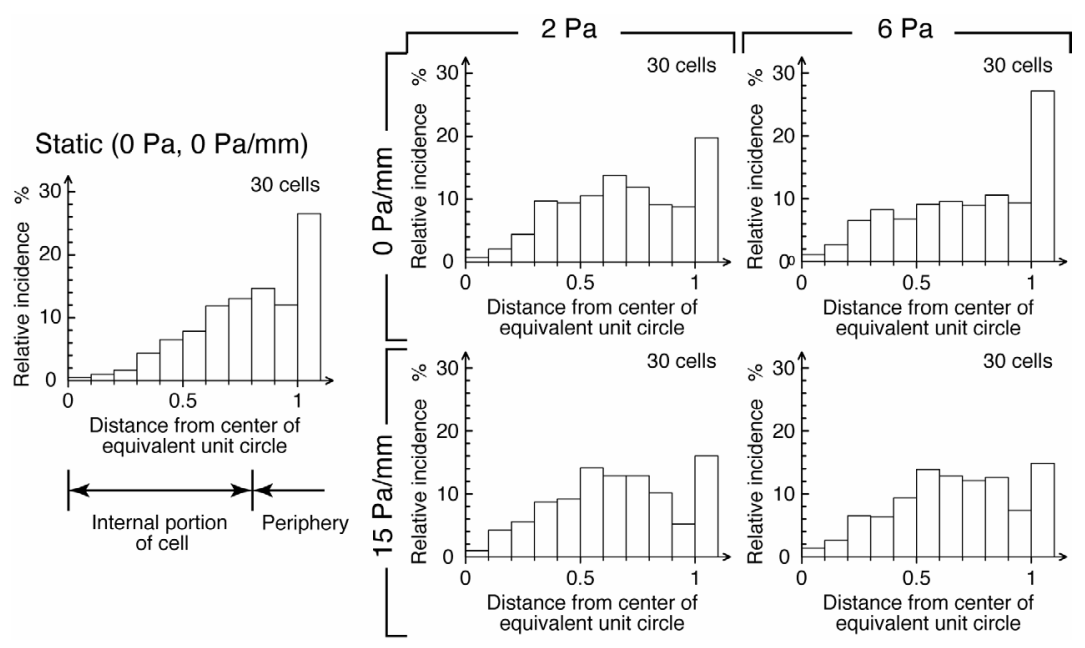

Fig. 5 Relative incidence of paxillin from the viewpoint of distance from the center of equivalent unit circle that indicates the EC outline.

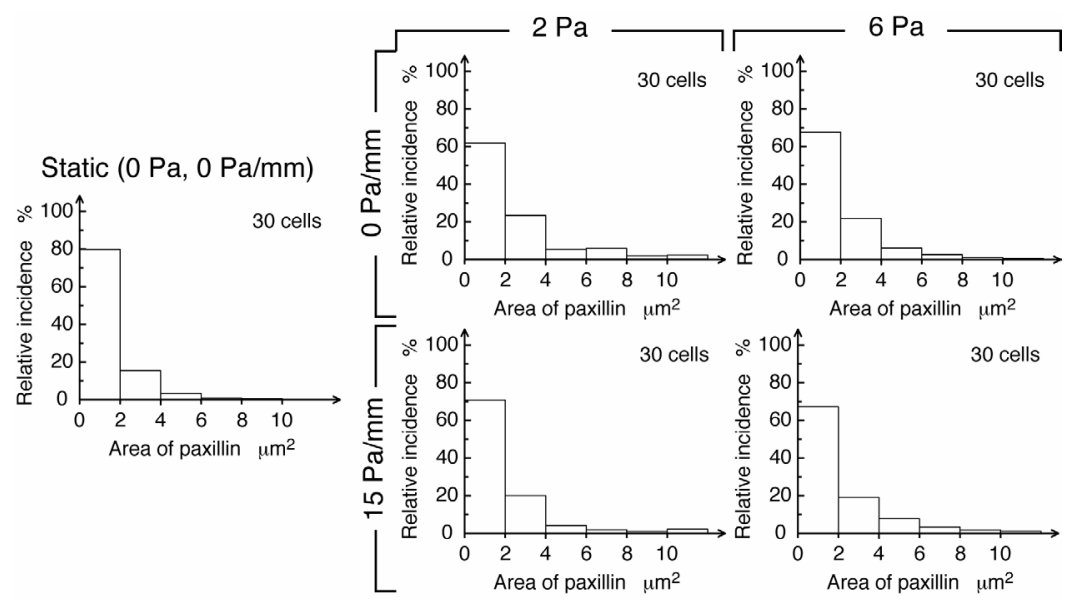

Fig. 6 Relative incidence of paxillin focusing on expression area of paxillin.

ECs exposed to SS without SSG was localized in the cell periphery. On closer inspection, paxillin was expressed in upstream and downstream in flow. ECs exposed to SS with SSG had the tendency to express paxillin not only in the periphery but also in the interior portion.

Tendency in areas of the expressed paxillin was similar, regardless of whether ECs were exposed to SS with or without spatial gradient. The areas of paxillin in the cells exposed to flow tended to increase in comparison with static condition, as illustrated in Fig. 6. Under the static condition, ECs had little expression of paxillin with area of more than $6 \mu^{2}$. Focusing on paxillin with the large area, namely the area is larger than $6 \mu \mathrm{m}^{2}$, we evaluated localization of the paxillin in the cell (Fig. 7). When ECs were exposed to SS with no spatial gradient, the paxillin with large area was commonly expressed in the cell periphery. In contrast, the cells exposed to flow with spatial gradient had a tendency to express the large paxillin in the interior portion.

\section{Discussion}

In the present study, we developed the D chamber that can generate uniform spatial SS gradient to evaluate effects of SSG itself on the cell morphology. The performance about linear increase in SS, namely constant SSG, was confirmed based on the CFD simulations. 


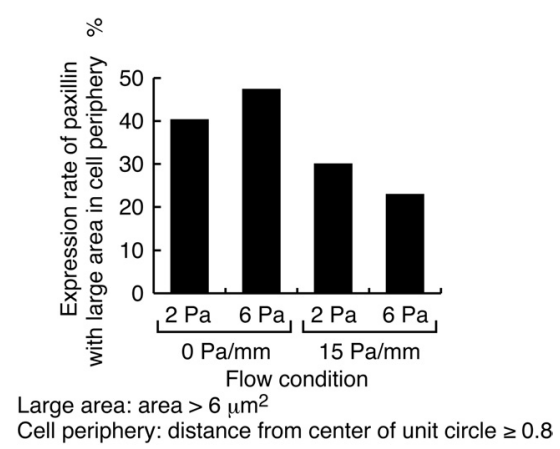

Fig.7 Expression rate of paxillin with large area $\left(>6 \mu \mathrm{m}^{2}\right)$ in cell periphery (distance from the center of unit circle $\geq 0.8$ ).

Although Dolan et al. ${ }^{(8)}$ manufactured the flow chamber with the channel similar to the D chamber, local increase in SSG and spike in SS were observed in upstream and downstream sides, respectively, and they could not generate the constant SSG condition. These problems were not found in our flow chamber. Difference in pressure between upstream and downstream in flow chamber was up to $3 \mathrm{kPa}(22.5 \mathrm{mmHg})$. Because this pressure value is lower than that in the previous studies about responses of ECs to hydrostatic pressure ${ }^{(14,15)}$, pressure is considered to have an insignificant effect on the cell morphology in the present experiments.

We clearly found the effect of combined SS and uniform SSG on EC response to fluid flow. After exposure to SS without spatial gradient for 24 hours, ECs elongated and oriented in the direction of flow. Actin filaments in the cell periphery developed and became thick along the direction of flow. In contrast, cells did not show obvious elongation, orientation, and development of thick actin filaments under the condition of SS with uniform SSG. Instead, thin and short filaments formed in the interior portion of the EC. The results in the present study correspond to the report of endothelial morphological responses under the combination of SS and variable SSG ${ }^{(7)}$. Intracellular signaling activated by SSG may inhibit the formation and development of thick actin filaments. As a result, it is considered that thin and short filaments formed in the interior portion of the EC. Therefore, these results suggest that cytoskeletal structures changed in response to the spatial gradient of SS.

Many studies have reported the mechanisms about sensing and converting SS into intracellular biochemical signals. Focal adhesion complex ${ }^{(16)}$, platelet-endothelial cellular adhesion molecules (PECAM)-1 ${ }^{(17)}$ are now thought as mechano-sensing molecules to SS. On the other hand, several studies have reported that difference in forces acting on intercellular junctions between the neighboring ECs along the direction of flow is important for sensing SSG. Ueki et al. ${ }^{(18)}$ have found that tensile force applied to intercellular junctions by using a microneedle causes recruitment of activated Src homology 2-containig tyrosine phosphatase-2 (SHP-2) binding to an intercellular adhesion molecule PECAM-1. This result suggests that ECs can sense SSG if SSG causes difference in forces on cell junctions. However, force acting on intercellular junctions by flow exposure is considered to be much smaller than that in the direct tensile experiment by the microneedle. In addition, because changes in SSG exists in the flow channel of T-chamber used in previous studies, big difference in forces acting on cell junction may occur in comparison with the condition of uniform SSG. When effects of SSG pure and simple on ECs' morphology are evaluated, difference in forces on a cell junction can contribute less to sensing of SSG. In this study, we thus focused on paxillin, which is a focal adhesion-associated protein. Under the condition of SS without SSG, paxillin localized in the periphery of the EC, and aggregation of paxillin occurred in relatively large numbers in the cell periphery. The previous studies have also reported that SS provoked disaggregation and redistribution of 
focal adhesion in the interior portion of a cell, and focal adhesion localized in the cell periphery ${ }^{(19)}$. However, paxillin was expressed not only in the cell periphery but also in the interior portion of the cell in the EC exposed to SS with its uniform SSG. Aggregation of paxillin tended to occur more commonly in the interior portion of the cell. Therefore, the spatial gradient of SS could inhibit redistribution of focal adhesion.

Endothelial cells are known to regulate cell migration and focal adhesion turnover by phosphorylation of 31st tyrosine residue to N-terminal (Tyr31) in paxillin ${ }^{(20)}$. When ECs, into which paxillin Y31A mutants were introduced, were exposed to SS of $1.2 \mathrm{~Pa}$ for 12 hours, paxillin Y31A mutants did not localize in the cell periphery and were distributed in the cell interior ${ }^{(20)}$. It was suggested that paxillin in ECs exposed to SS was not redistributed and did not aggregate in the cell periphery by inhibiting phosphorylation of Tyr31 in paxillin. This phenomenon is similar to distributions of paxillin in ECs under the condition of SS with uniform SSG. Therefore, SSG could have the potential to inhibit phosphorylation of Tyr31 in paxillin. Alternatively, SSG might be considered to suppress activation of FAK or proto-oncogene tyrosine-protein kinase Src, which cause phosphorylation of Tyr31 in paxillin ${ }^{(21)}$.

The spatial gradient of SS has an important role in suppressing development of actin filaments and redistribution of focal adhesion, which are associated with morphological changes in ECs. However, it still remains unclear whether signal transduction of SSG via focal adhesion or intercellular adhesion molecules dominantly acts on endothelial morphology. The detail about signal transduction of SSG will be investigated in future studies.

\section{Conclusion}

In the present study, we developed the novel flow chamber to evaluate effects of SSG on morphological responses of ECs to fluid flow. ECs' responses to the combination of SS and uniform SSG were investigated using the developed chamber. ECs maintained a confluent monolayer under SS of 2 and 6 Pa with SSG of 0 and $15 \mathrm{~Pa} / \mathrm{mm}$. ECs elongated and aligned parallel to the direction of flow with development of thick actin filaments and localization of paxillin in the cell periphery after exposure to SS without SSG for 24 hours. In contrast, the ECs exposed to SS with SSG did not elongate and orient in the direction of flow, development of thick actin filaments and the localization of paxillin were not confirmed. These results suggested that SSG suppresses formations of actin filaments and redistribution of focal adhesion, resulting in the inhibition of EC morphological changes in response to flow.

\section{Acknowledgment}

The present study was supported in part by Grants-in-Aid for Scientific Research from the Ministry of Education, Culture, Sports, Science and Technology (MEXT) of Japan (No. 20001007).

\section{References}

(1) Kataoka, N., Ujita, S., Kimura, K. and Sato, M., The morphological responses of cultured bovine aortic endothelial cells to fluid-imposed shear stress under sparse and colony conditions, JSME International Journal, Series C, Vol.41, No.1 (1998), pp.76-82.

(2) Malek, A. M., Alper, S. L. and Izumo, S., Hemodynamic shear stress and its role in atherosclerosis, Journal of the American Medical Association, Vol.282, No.21 (1999), pp.2035-2042.

(3) Davies, P. F., Flow-mediated endothelial mechanotransduction, Physiological Reviews, 
Vol.75, No.3 (1995), pp.519-560.

(4) Sato, M., Suzuki, K., Ueki, Y. and Ohashi, T., Microelastic mapping of living endothelial cells exposed to shear stress in relation to three-dimensional distribution of actin filaments, Acta Biomaterialia, Vol.3, No.3 (2007), pp.311-319.

(5) Zaidel-Bar, R., Kam, Z. and Geiger, B., Polarized downregulation of the paxillin-p130CAS-Rac1 pathway induced by shear flow, Journal of Cell Science, Vol.118, No.17 (2005), pp. 3997-4007.

(6) Szymanski, M. P., Metaxa, E., Meng, H. and Kolega, J., Endothelial cell layer subjected to impinging flow mimicking the apex of an arterial bifurcation, Annals of Biomedical Engineering, Vol.36, No.10 (2008), pp.1681-1689.

(7) Sakamoto, N., Saito, N., Han, X., Ohashi, T. and Sato, M., Effect of spatial gradient in fluid shear stress on morphological changes in endothelial cells in response to flow, Biochemical and Biophysical Research Communications, Vol.395, No.2 (2010), pp.264-269.

(8) Dolan, J. M., Meng, H., Singh, S., Paluch, R. and Kolega, J., High fluid shear stress and spatial shear stress gradients affect endothelial proliferation, survival, and alignment, Annals of Biomedical Engineering, Vol.39, No.6 (2011), pp.1620-1631.

(9) Bacabac, R. G., Smit, T. H., Cowin, S. C., Van Loon, J. J., Nieuwstadt, F. T., Heethaar, R. and Klein-Nulend, J., Dynamic shear stress in parallel-plate flow chambers, Journal of Biomechanics, Vol.38, No.1 (2005), pp.159-167.

(10) Meng, H., Wang, Z., Hoi, Y., Gao, L., Metaxa, E., Swartz D. D. and Kolega, J., Complex hemodynamics at the apex of an arterial bifurcation induces vascular remodeling resembling cerebral aneurysm initiation, Stroke, Vol.38, No.6 (2007), pp.1924-1931.

(11) Sakamoto, N., Ohashi, T. and Sato, M., Effect of magnetic field on nitric oxide synthesis of cultured endothelial cells, International Journal of Applied Electromagnetics and Mechanics, Vol.14, No.1-4 (2002), pp.317-322.

(12) Oshima, M., Image-based simulation of blood flow for cerebrovascular disorders, Journal of Japan Society of Fluid Mechanics, Vol.21, No.2 (2002), pp.122-128.

(13) Castro, M. A., Putman, C. M., Sheridan, M. J. and Cebral, J. R., Hemodynamic patterns of anterior communicating artery aneurysms: a possible association with rupture, American journal of neuroradiology, Vol.30, No.2 (2009), pp.297-302.

(14) Ohashi, T., Sugaya, Y., Sakamoto, N. and Sato, M., Hydrostatic pressure influences morphology and expression of VE-cadherin of vascular endothelial cells, Journal of Biomechanics, Vol.40, No.11 (2007), pp.2399-405

(15) Müller-Marschhausen, K., Waschke, J. and Drenckhahn, D., Physiological hydrostatic pressure protects endothelial monolayer integrity, American Journal of Physiology. Cell Physiology, Vol.294, No.1 (2008), pp.C324-C332.

(16) Wang, Y., Miao, H., Li, S., Chen, K. D., Li, Y. S., Yuan, S., Shyy, J. Y. and Chien, S., Interplay between integrins and FLK-1 in shear stress-induced signaling, American journal of physiology. Cell physiology, Vol.283, No.5 (2002), pp.C1540-C1547.

(17) Tzima, E., Irani-Tehrani, M., Kiosses, W. B., Dejana, E., Schultz, D. A., Engelhardt, B., Cao, G., DeLisser, H. and Schwartz, M. A., A mechanosensory complex that mediates the endothelial cell response to fluid shear stress, Nature, Vol.437, No.7057 (2005), pp.426-431.

(18) Ueki, Y., Sakamoto, N., Ohashi, T. and Sato, M., Morphological responses of vascular endothelial cells induced by local stretch transmitted through intercellular junctions, Experimental Mechanics, Vol.49, No.1 (2009), pp.125-134.

(19) Li, S., Butler, P., Wang, Y., Hu, Y., Han, D., Usami, S., Guan, J. and Chien, S., The role of the dynamics of focal adhesion kinase in the mechanotaxis of endothelial cells, Proceedings of the National Academy of Sciences of the United States of America, Vol.99, No.6 (2002), pp.3546-3551.

(20) Mattiussi, S., Matsumoto, K., Illi, B., Martelli, F., Capogrossi, M. C. and Gaetano, C., 
Papilloma protein E6 abrogates shear stress-dependent survival in human endothelial cells: Evidence for specialized functions of paxillin, Cardiovascular Research, Vol.70, No.3 (2006), pp.578-588.

(21) Brown, M. C. and Turner, C. E., Paxillin: adapting to change, Physiological Reviews, Vol.84, No.4 (2004), pp.1315-1339. 